\title{
Atomic-resolution STEM Analysis of Nanoparticle During Electrocatalytic Reactions
}

Jinglong Guo ${ }^{1}$, Xue Rui ${ }^{1}$, Dongyoung Chung ${ }^{2}$, Pietro Papa Lopes ${ }^{2}$ and Robert Klie ${ }^{1}$

${ }^{1}$ University of Illinois at Chicago, Chicago, Illinois, United States, ${ }^{2}$ Argonne National Laboratory, Lemont, Illinois, United States

The oxygen evolution reaction (OER) is an important process for many energy storage applications, such as rechargeable metal-air battery or for the production of hydrogen. Good OER electro-catalysts, including metal oxide nanoparticles, allow for anionic redox of oxygen, both in bulk and at the particle surface [1, 2], but may lead to significant dissolution and degradation of catalyst. In perovskite oxides of the form $\mathrm{ABO}_{3}$, it has been previously demonstrated that enhancing the hybridization between the $\mathrm{O} 2 \mathrm{p}$ and transition-metal $3 d$ orbitals can enhance the OER activity.[3] Doping the lanthanide $A$-site, as in Sr-doped $\mathrm{LaCoO}_{3}$ (LSCO), has been widely utilized to affect the metal-oxygen covalence. With increasing the $\mathrm{Sr}^{2+}$ substitution of $\mathrm{La}^{3+}$, the $\mathrm{O} 2 p$ band will move toward the Co $3 d$ band with decreasing Femi level, which can trigger the OER on lattice oxygen sites.[4] Meanwhile, Fe impurities in the $\mathrm{KOH}$ electrolyte have been found to significantly increase the activity of Ni-based perovskite oxide electrocatalysts.[5] Therefore, in this work we evaluate the role of Fe impurities together with Sr-doping on LaCoO3 based materials on their activity and stability for OER in alkaline electrolyte.

This contribution will utilize a combination of scanning transmission electron microscopy (STEM) imaging, electron energy loss spectrum (EELS) and energy dispersive x-ray (EDX) to examine the effects of electrochemical cycling of $\mathrm{La}_{1-x} \mathrm{Sr}_{\mathrm{x}} \mathrm{CoO}_{3}$ catalysts in an ultrapure $\mathrm{KOH}$ electrolyte and after the addition Fe ions. Specifically, we will compare the chemical composition, atomic structure and Co valence states of pristine and cycled samples. These studies are carried out in the JEOL-JEM ARM200CF equipped with Gatan Quantum Continuum spectrometer and Oxford X-max 100TLE windowless SDD Xray detector. To gain the capability to resolve the effect of electrochemical cycling on the particle evolution, our analysis is conducted on the same particles first on pristine and then cycled materials (Identical Location; ILTEM). This will enable us to correlate the changes in the atomic and electronic structures at atomic-resolution using the combination of STEM imaging, electron energy-loss spectroscopy (EELS) and energy-dispersive X-ray spectroscopy imaging (XEDS).

In addition to the $\mathrm{La}_{1-\mathrm{x}} \mathrm{Sr}_{\mathrm{x}} \mathrm{CoO}_{3}$ catalysts, we also studied MnWO particles decorated with Ir catalyst nanoparticles for OER in acidic media using the ILTEM characterization approach. Here, the catalyst particles were deposited on carbon-coated $\mathrm{Au}$ grid and studied first in their pristine form, after electrochemical cycling, and then separately, on particles cycled in a larger electrocatalytic reactor. Figure 1 shows atomic resolution structures of pristine MnWO particles and MnWO particles covered with Iridium nanoparticles. Atomic structure models are shown in inserts respectively. The Ir nanoparticles can be found on the MnWO surfaces (indicated by arrows in Figure 1 (b)). Figure 2 shows the same MnWO particle before (Figure2 a) and after cycling (Figure2b). After cycling, significant changes in the MnWO particles' morphology and the Ir coverage were observed. The dark circular area, which is due to a void within the MnWO particle, serves as a fiduciary marker, and suggests an expansion of the void during cycling, potentially due to MnWO particles dissolution. The decrease of Ir nanoparticle on the surface of MnWO particles was also observed. The results will be combined with first principles modeling to 
demonstrate the effects of surface composition and other structural evolution following the activity of the OER catalysts. [6]

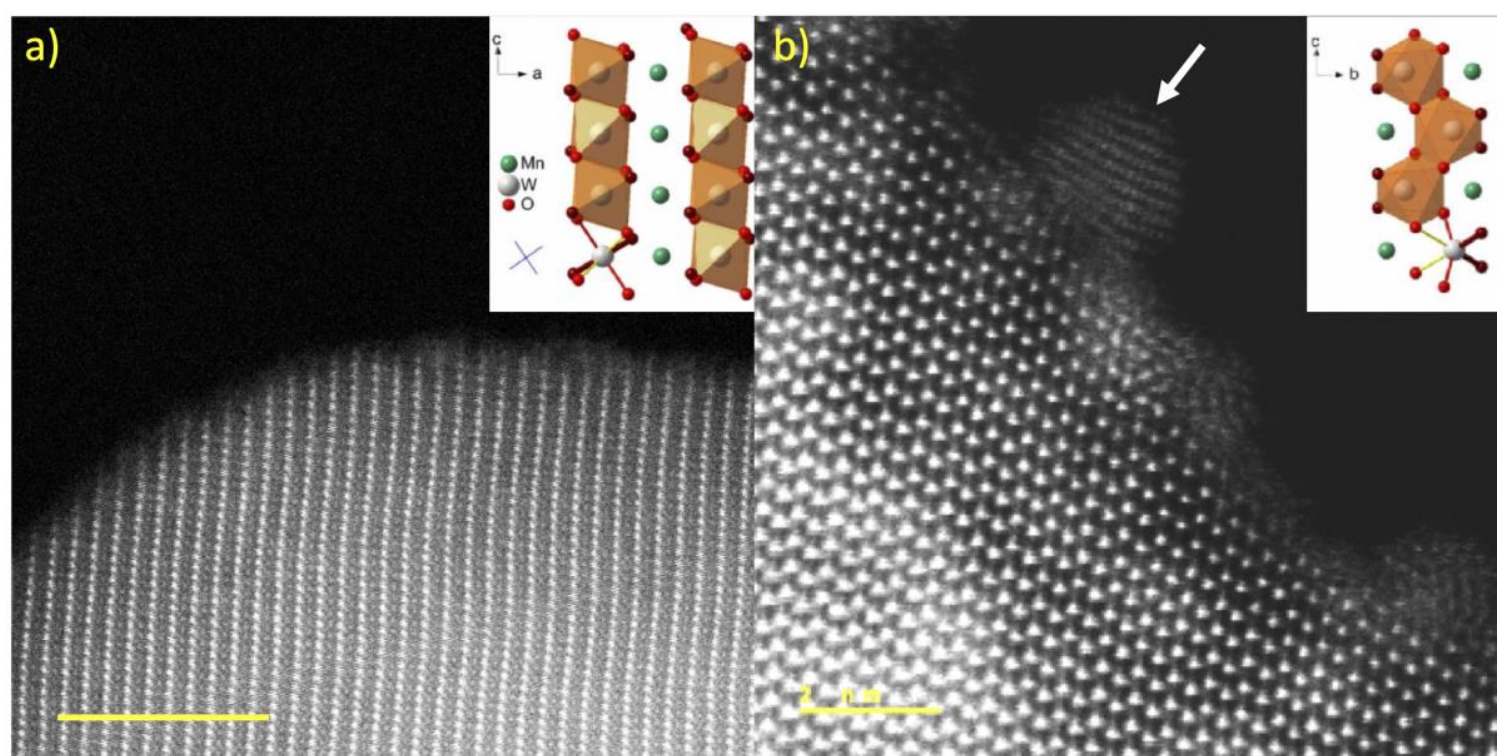

Figure 1. Atomic resolution HAADF images of MnWO particles a) with and b) without Ir nanoparticle. One of an Ir nanoparticles is marked by the arrow in b).

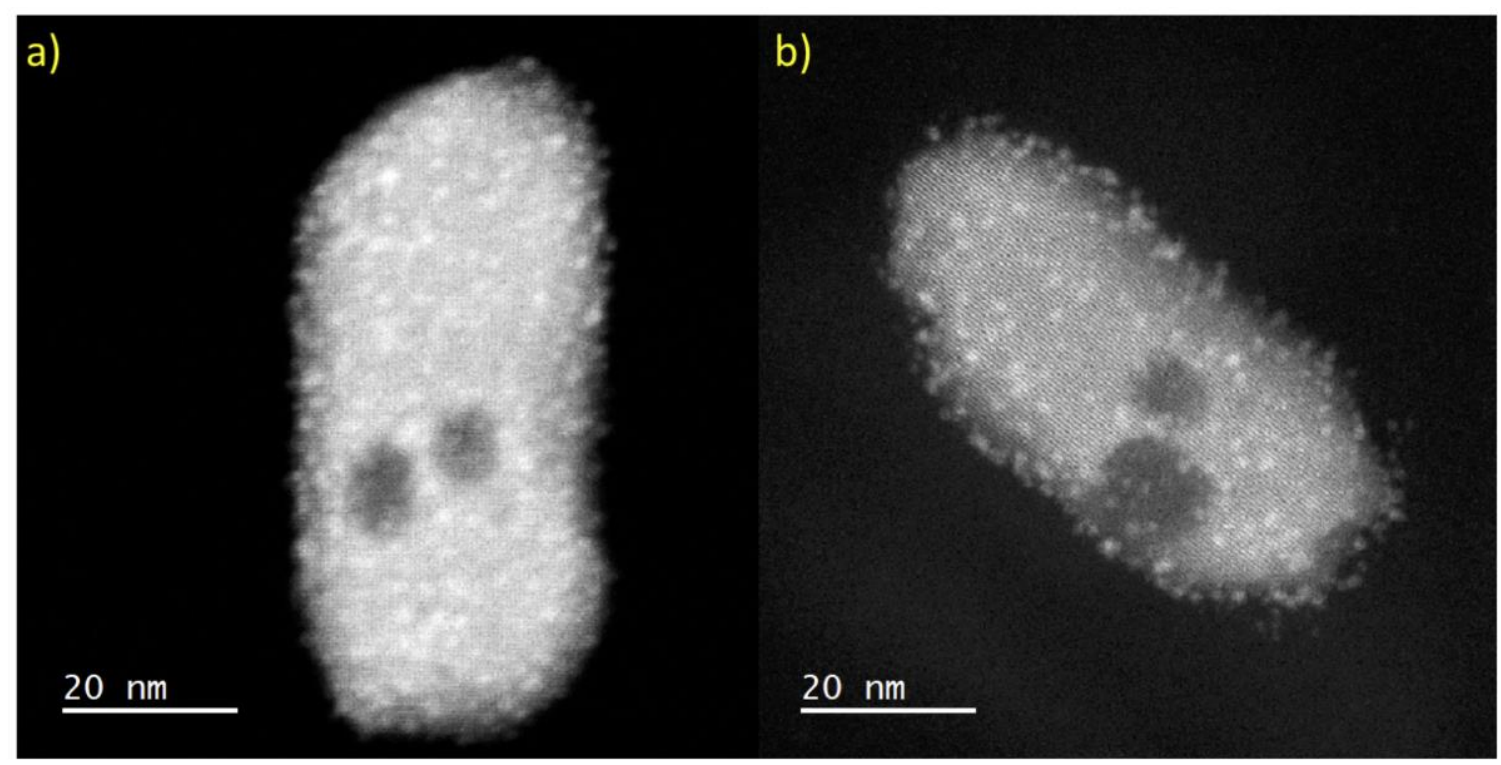

Figure 2. a) HAADF STEM image of Ir:MnWO particle before cycling. b) The same particle after cycling.

\section{References}

[1] E Fabbri et al., Nature Materials 16 (2017), p. 925.

[2] X Cheng et al., Chemistry of Materials 27 (2015), p. 7662.

[3] JT Mefford et al., Nat Commun 7 (2016), p. 11053.

[4] A Grimaud et al., Nature Chemistry 9 (2017), p. 828.

[5] L Trotochaud et al., Journal of the American Chemical Society 136 (2014), p. 6744.

[6] This work is supported by National Science Foundation (No. DMR-1831406). 